UNDERGRADUATE RESEARCH IN NATURAL AND CLINICAL SCIENCE AND TECHNOLOGY (URNCST) JOURNAL Read more URNCST Journal articles and submit your own today at: https://www.urncst.com

\title{
The 4th Annual CCNM Research Day: Student Research \& Innovation in Naturopathic Medicine
}

Monique Aucoin, BMSc ND [1]*, Valentina Cardozo, BSc MSc [1],

Christine Chung, CCNM Student [1], Kieran Cooley BSc ND [1-3]

[1] Canadian College of Naturopathic Medicine, Toronto, Ontario, Canada M2K 1E2

[2] University of Technology, Australian Research Center in Complementary and Integrative

Medicine, Ultimo, New South Wales, Australia

[3] Pacific College of Oriental Medicine, San Diego, California, United States of America

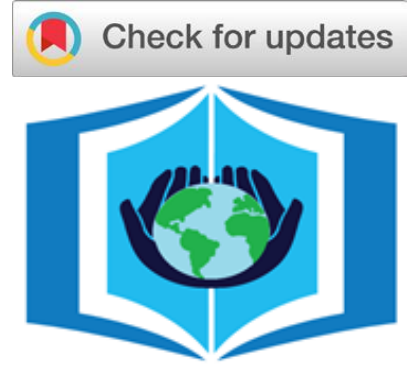

URNCST Journal

"Research in Earnest"

*Corresponding Author: maucoin@ccnm.edu

\begin{abstract}
The following are abstracts from the research competition at the 4th annual CCNM Research Day hosted by the Canadian College of Naturopathic Medicine in Toronto, ON, Canada. The conference celebrates high quality student-faculty research collaborations, showcased as poster presentations.
\end{abstract}

Keywords: naturopathy; research; naturopathic medicine; complementary medicine; integrative medicine; undergraduate research competition; innovation; mentors

Table of Contents

Poster Presentation Abstracts.

pg. A01-A09

Constitutional hydrotherapy: Can it improve headache and mood? A case report

pg. A02-A02

Oral supplementation of myo-inositol in individuals with polycystic ovarian syndrome (PCOS) and infertility: A narrative review

Minimizing isotretinoin side effects with concurrent oral supplementation: A narrative review

pg. A02-A03

Potential therapeutic use of curcumin and ginseng following mild traumatic brain injury: A narrative

pg. A03-A03

review

No improvement in disclosure of natural health product use to primary care medical doctors in the last 15 Years: A survey of naturopathic patients.

pg. A03-A04

Investigating natural therapies for dysmenorrhea: A narrative review

Acupuncture as a treatment for depression in cancer patients: A narrative review

Are we supporting the well-being of medical students? A scoping review.....

Effects of short term fasting on cancer treatment: A narrative review

Different health professionals' uses of strontium: A narrative review

The potential therapeutic effect of topical cannabinoids in psoriasis: A review of the literature

The potential therapeutic effect of topical cannabinoids in atopic dermatitis: A review of the literature

Dietary counseling in schizophrenia spectrum disorders: Creation and evaluation of an evidence-

informed psychoeducational tool.....

Patterns of integrative medicine (IM) use and its effects on quality of life of patients undergoing

radiotherapy: A protocol.

pg. A04-A04

pg. A04-A05

pg. A05-A05

pg. A05-A06

pg. A06-A06

pg. A06-A07

pg. A07-A07

pg. A07-A08

pg. A08-A08

The relationship between serum vitamin D status and breast cancer risk and occurrence: A narrative

pg. A08-A08 review

Note: Research posters associated with this conference abstract book are available as a supplementary file. 
UNDERGRADUATE RESEARCH IN NATURAL AND CLINICAL SCIENCE AND TECHNOLOGY (URNCST) JOURNAL Read more URNCST Journal articles and submit your own today at: https://www.urncst.com

\section{$\underline{\text { Poster Presentation Abstracts }}$}

Constitutional hydrotherapy: Can it improve headache and mood? A case report Maryam Yavari, CCNM Student [1], Afsoun Khalili, ND [1] [1] Canadian College of Naturopathic Medicine, Toronto, Ontario, Canada

Background: Constitutional hydrotherapy, which applies thermal alteration and mild electrical stimulation, has been used in naturopathic medicine for a long time. This is the first case report on the effects of this therapy on headache and mood.

Case Presentation: A 59 year old female was admitted to the Robert Schad Naturopathic Clinic with anxiety and nervousness. We performed two sessions of constitutional hydrotherapy in 2 weeks. Treatment included five phases of hydrotherapy as follows: hot (5 min), cold + electrical stimulation (10 min), dry+ electrical stimulation (10 min), hot (5 min), and cold (5 min). During therapy, the patient was very relaxed, in a very emotional, joyful mood and was constantly tearing. She felt the therapy was "releasing her suppressed emotions" and making her very light. The most significant side benefit of this therapy was that the patient had a headache before the first session and after treatment, the pain was completely resolved. The patient also reported having more energy after the treatment. The patient filled the English version of multi-dimensional mood state questionnaire with 6-point Likert scales as an assessment in three dimensions of good/bad mood, awake/tired and calm/nervous. The scores for the good/bad mood dimension were 31, 58, 60; for the awake/tired section were 31,52, 60; and for the calm/nervous section were 27, 59, 60; at baseline, between the sessions, and after the treatment respectively. The patient expressed that "the therapy has been amazing for me and improved drastically my thinking paradigm and mental health".

Discussion: Despite the long history of constitutional hydrotherapy, mood/emotional disorders and headache are not listed as traditional indications for this method. The only scientific evidence available about this therapy are studies that support its effects on physical functioning and quality of life. The mechanisms of observed effect in this study need further evaluation but may include effects on thermal hemostasis, hydrostatic shifting, integumentary system, blood circulation, neurotransmitters, and autonomic nervous system regulation.

Conclusions: Our study supports the use of constitutional hydrotherapy in altering emotional states, reducing anxiety and improving mood and headache. Considering the limitations of this study (including lack of a control group and a small sample size) we suggest that additional research is needed in order to support the therapeutic benefits of constitutional hydrotherapy. The authors declare there are no conflicts of interest and they have not received financial support for this research.

Oral supplementation of myo-inositol in individuals with polycystic ovarian syndrome (PCOS) and infertility: A narrative review

Daiana Pogacean, HBSc, CCNM Student [1], Zeynap Uraz, ND [1]

[1] Canadian College of Naturopathic Medicine, Toronto, Ontario, Canada

Background: PCOS is the most common endocrine disorder in menstruating individuals of reproductive age; the prevalence in Canada is approximately 6.5-8\%. Insulin resistance is a key metabolic component of PCOS that contributes to symptoms and myo-inositol (MI) has been shown to increase insulin sensitivity. This review aimed to identify key studies demonstrating outcomes related to ovulation and pregnancy rates in in vitro-fertilization (IVF) and intrauterine insemination (IUI) when supplementing with MI.

Methods: This narrative review was conducted on PubMed with the search terms: inositol, PCOS and, female, and "clinical trial" was selected as an additional filter. This search yielded a total of 44 unique studies up to January 2020 . We excluded reviews, as well as studies comparing MI to metformin or to D-chiro inositol. A total of four randomized controlled studies were included in this review.

Results: All studies included in this narrative review demonstrate positive reproductive outcomes in individuals who supplemented with MI. The first study found that the MI group had a significantly increased frequency of ovulation compared with the placebo group. The second found that total FSH dose and cycle duration were significantly lower and clinical pregnancy rates were higher in the MI group during controlled ovarian hyperstimulation and IUI treatment. The third study found that those in the MI intervention group had a reduced number of immature oocytes, greater number of follicles with diameter $>15 \mathrm{~mm}$, and a higher number of oocytes at retrieval during IVF. The fourth study confirmed these findings with the MI group exhibiting higher biochemical pregnancy rate, higher number of large-sized follicles (>16mm), and oocytes of greater quality during IVF. 
UNDERGRADUATE RESEARCH IN NATURAL AND CLINICAL SCIENCE AND TECHNOLOGY (URNCST) JOURNAL Read more URNCST Journal articles and submit your own today at: https://www.urncst.com

Conclusion: These preliminary small studies show that individuals who supplement with MI can increase rates of clinical pregnancy and ovulation frequency, and support IVF by decreasing the mean number of immature oocytes, increasing the number of follicles with a greater diameter, and retrieving oocytes of higher quality. MI exhibits a promising therapeutic role in this population and further studies should investigate its efficacy of patient-important outcomes such as live birth for women with PCOS who are experiencing infertility.

The authors have no conflict of interest to declare.

Minimizing isotretinoin side effects with concurrent oral supplementation: A narrative review

Jessica Maloh, BSc, CCNM Student [1], Joy Dertinger, ND [1]

[1] Canadian College of Naturopathic Medicine, Toronto, Ontario, Canada

Introduction: Acne vulgaris is a common skin condition associated with scarring, dyspigmentation and low self-esteem. Isotretinoin is a common and effective treatment option. However, adverse effects include cheilitis, xerosis, myalgia, elevated liver enzymes, changes to triglyceride levels and nutrient levels, such as folic acid. This narrative review explores the concurrent use of oral supplements in minimizing isotretinoin's adverse effects.

Methods: The terms [(isotretinoin OR accutane) AND (prevent OR reduce OR change) AND side effects]; [omega-3 AND isotretinoin]; AND [(supplement OR supplementation) AND (accutane OR isotretinoin)] were used in PubMed. This search yielded a total of 212 studies (up to January, 2020). Only studies with humans using isotretinoin for acne were included. Exclusion criteria consisted of additional pharmaceuticals, topical interventions and injections. A total of 10 studies were included.

Results: Omega-3s: A longitudinal survey found patients not using concurrent omega-3 had greater increases in triglycerides (TG) after Iso use $(\mathrm{P}=0.04)$. One RCT found patients taking fish oil with Iso had lower TG and cholesterol levels (both $\mathrm{P}<0.05)$. Another found concurrent omega-3s resulted in less frequent dry lips $(\mathrm{P}=0.03)$, nose $(\mathrm{P}=0.01)$, skin $(\mathrm{P}=0.003)$, and eyes $(\mathrm{P}=0.046)$. Evening Primrose Oil (EPO): Based on a pilot study, EPO alongside Iso lowers cheilitis $(\mathrm{P}=0.035)$ and TEWL ( $\mathrm{P}=0.043)$, while significantly increasing patient satisfaction. Vitamin E: The evidence is mixed- one RCT showed reduction in cheilitis $(\mathrm{P}=0.037)$, while another showed significant increases in the severity of side effects, and another, no statistical differences. Folic acid \& B12: An RCT found Iso with folic acid and B12 decreased homocysteine (P=0.0004), while increasing folic acid $(\mathrm{P}=0.0026)$ and $\mathrm{B} 12(\mathrm{P}=0.0002)$. L-Carnitine: A controlled trial found L-carnitine used with Iso results in disappearance of muscular side effects, while normalizing liver enzymes and L-carnitine levels. Combination Supplement: An RCT using gamma linolenic acid, vitamin E, vitamin C, B-carotene, coQ10, and Vitis vinifera alongside Iso showed reductions in sebum production rate, increases in hydration, less erythema, and greater adherence to Iso (P<0.0001).

Conclusion: The use of Iso alongside oral omega-3s, EPO, folic acid and vitamin B12, L-carnitine, and a combination supplement can decrease Iso adverse effects. However, the effect of oral vitamin E is unclear. Further research is needed to replicate these findings, and to determine the most effective treatment dosages.

The authors declare that they have no conflicts of interest.

Potential therapeutic use of curcumin and ginseng following mild traumatic brain injury: A narrative review Michelle Malik, CCNM Student [1], As'ad Hamid, ND [1]

[1] Canadian College of Naturopathic Medicine, Toronto, Ontario, Canada

Background: Mild traumatic brain injury (MTBI) resulting from a force applied to the brain or body can result in a wide range of cognitive, neurological and systemic symptoms. In cases of recurrent injury, untreated MTBI can progress to serious neurodegenerative disease and result in mortality. The incidence of MTBI in Canada is 210,000 cases annually, and $1.2 \%$ of Ontario's population experiences MTBI each year. Youth and athletes are at highest risk, and repetitive MTBI is a major contributor to mortality world-wide. In this narrative review we researched the phytochemical properties of the specific herbs, ginseng and curcumin, and their potential therapeutic use for treatment of MTBI. Ginseng and curcumin were chosen due to the number of studies performed, compared to other herbs, that investigated their use and mechanisms of action in improving symptoms following MTBI.

Methods: Cochrane and PubMed databases were searched using the terms; "traumatic brain injury", "natural treatment", "ginseng", "curcumin", and "cognition". The literature search was restricted to years 2010-2020 and only included articles that focussed on TBI treatment. Treatments of stroke and other brain-related health issues were excluded.

Results: A total of nine papers with various animal-model studies were reviewed. Our search did not identify any human studies performed in this area of research. Six of the nine studies examined the effects of curcumin on induced MTBI in rats. Curcumin showed neurological benefits for treatment through counteraction of spinal cord deficits, improving hippocampal

Aucoin et al. | URNCST Journal (2020): Volume 4, Issue 3

DOI Link: https://doi.org/10.26685/urnest.186

Page A3 of A10 
UNDERGRADUATE RESEARCH IN NATURAL AND CLINICAL SCIENCE AND TECHNOLOGY (URNCST) JOURNAL Read more URNCST Journal articles and submit your own today at: https://www.urncst.com

learning and fine motor coordination. Additionally, curcumin was shown to reduce oxidative stress and decrease inflammation in rat neuronal cells through potential use of the TLR4 pathway. Moreover, six of the nine studies reviewed investigated the effects of ginseng on induced MTBI in rats. Ginseng showed protective effects against fatal ischemic damage in neuronal cells, in addition to cell proliferation and enhanced survival rate of newborn cells. Interestingly, a proposed reason for the neuronal protection discovered is due to the compound ursolic acid which is present in both, ginseng and curcumin. Ursolic acid aids to decrease oxidative stress and cytokines promoting inflammation.

Conclusions: Curcumin and ginseng both show efficacy for neuroprotection and thus may be beneficial for MTBI treatment in animal models. Further combination research and human trials should be performed to evaluate the clinical efficacy of using ginseng and curcumin for treatment of MTBI.

The authors declare that they have no conflicts of interest.

No improvement in disclosure of natural health product use to primary care medical doctors in the last 15 Years: A survey of naturopathic patients

Jeremy Ng, MSc [1], Anna Garber, CCNM Student [2], Michelle Luong, BSc [1], Kieran Cooley, ND [2-4], Jason Busse, PhD [1]

[1] Department of Health Research Methods, Evidence, and Impact, Faculty of Health Sciences, McMaster University, Hamilton, Ontario, Canada

[2] Canadian College of Naturopathic Medicine, Toronto, Ontario Canada

[3] Australian Research Center in Complementary and Integrative Medicine, University of Technology, Ultimo, New South

Wales, Australia

[4] Pacific College of Oriental Medicine, San Diego, California, United States of America

Background: The use of natural health products (NHPs) is common in North America. In 2003, we found that 42\% of NHP users had not disclosed this information to their primary care medical doctors (MDs). We repeated our survey in 2018/2019 to explore if the rate of NHP use disclosure had improved.

Methods: From November, 2018 to February, 2019, a 21-item survey about NHP use and disclosure was administered to adult patients who visited the Robert Schad Naturopathic Clinic in Toronto, Canada.

Results: Almost all patients surveyed were using NHPs (99\%), and 46\% were using NHPs and prescription medication concurrently. Consistent with our 2003 findings, $42 \%$ of respondents who used NHPs did not disclose this information to their MD.

Conclusion: Disclosure of NHP use to MDs by naturopathic patients is limited and remained unchanged over the past 15 years. Future research should explore primary care MDs' hesitancy to inquire about patient NHP use.

The authors declare that they have no conflicts of interest. This work has been published, in full text elsewhere as: Jeremy Y. $\mathrm{Ng}$, Anna Garber, Michelle Luong, Kieran Cooley and Jason W. Busse, Complementary Therapies in Clinical Practice, 2020-05-01, Volume 39, Article 101106. https://doi.org/10.1016/j.ctcp.2020.101106

Investigating natural therapies for dysmenorrhea: A narrative review

Nadine Kexel, CCNM Student [1], Adam Gratton, ND [1]

[1] Canadian College of Naturopathic Medicine, Toronto, Ontario, Canada

Background: Primary dysmenorrhea is a condition characterized by abdominal and/or pelvic pain associated with menses. It affects more than half of menstruating Canadian women and 15\% are incapacitated for 1-3 days every cycle. Nonsteroidal anti-inflammatory drugs are often times used by this population in order to mitigate pain, and when this does not work, hormonal therapy is considered. These pharmaceutical options cause a number of adverse effects. Hormonal therapy in particular increases risk of breast cancer and infertility. Non-pharmacological therapies that pose less health risks have been explored, but are not regularly incorporated in the clinical setting. The purpose of this investigation was to analyze current evidence on dietary supplements that may effectively reduce symptoms of dysmenorrhea.

Methods: PICO criteria were defined prior to conducting the literature search: 1) the population had to include females with primary dysmenorrhea, 2) the interventions included any natural dietary supplement, 3) there study had to include a placebo control group and 4) Visual Analog Scale (VAS) had to be one of the outcome measures. In order to find research that matched these criteria, a literature search was conducted on the Dietary Supplement subset of the PubMed database and the human filter applied. 
UNDERGRADUATE RESEARCH IN NATURAL AND CLINICAL SCIENCE AND TECHNOLOGY (URNCST) JOURNAL Read more URNCST Journal articles and submit your own today at: https://www.urncst.com

Results: The literature search yielded a total of 417 studies of which ginger, vitamin D, and omega-3s/fish oil were found to be the only interventions with a minimum of two clinical trials pertaining to the treatment of dysmenorrhea. All eight studies using ginger, vitamin D or fish oil omega-3s as interventions to reduce the symptoms of primary dysmenorrhea showed a statistically significant improvement in VAS scores in comparison to the placebo. However, the magnitude of effect and dosages used varied between studies. Ginger doses were $1500 \mathrm{mg} /$ day, vitamin D 50,000-300,000 IU (single administration), and fish oil/omega-3s 300-755 mg/day.

Conclusion: Ginger, vitamin D and omega-3s/fish oil may be viable treatments and prophylactic options for females with dysmenorrhea. Additional studies with larger sample size are needed to strengthen the validity of these therapies and determine optimal dosage for positive clinical outcomes.

The authors declare that they have no conflicts of interest.

Acupuncture as a treatment for depression in cancer patients: A narrative review Erica Eckstrand, CCNM Student [1], Janis Li, ND [1]

[1] Canadian College of Naturopathic Medicine, Toronto, Ontario, Canada

Background: One in two Canadians are diagnosed with cancer in their lifetime and one in five of them will experience depression while living with cancer. Acupuncture is one of many naturopathic interventions that could benefit patients with cancer-related depression. This presentation reviews the current evidence on acupuncture to treat depression in cancer patients.

Methods: A search of peer-reviewed literature was conducted on PubMed with the search terms "acupuncture", "cancer" and "depression". The search was limited to articles between 2009-2019 and studies that used measurable depression scales.

Results: One randomized controlled trial, one observational data collection study and one literature review were used to review the efficacy of treating cancer patients with depression. The evidence found in these studies shows that acupuncture not only provides therapeutic benefit to cancer patients for a wide range of cancer-related physical symptoms such as nausea and vomiting; but it may also be an effective treatment for highly prevalent psychological symptoms in this population, including depression, anxiety and insomnia.

Conclusion: Acupuncture can relieve symptoms of depression in cancer patients to an even greater degree than conventional treatment (e.g. Fluoxetine) and alleviates other cancer-related symptoms as well, including insomnia, anxiety and pain.

The authors have no conflicts of interest to declare.

Are we supporting the well-being of medical students? A scoping review

Shakila Mohmand, CCNM Student [1], Imane Squalli Houssaini, CCNM Student [1], Sasha Monteiro, CCNM Student[1], Leslie Solomonian, ND [1]

[1] Canadian College of Naturopathic Medicine, Toronto, Ontario, Canada

Background: Medical students experience significant stress and mood impacts as a result of academic workload, financial loans, and uncertain career choices. Unresolved stress can result in impacted mood, burnout, physical impairments and increased risk of unethical behaviour. Studies have failed to establish distinct coping strategies to improve the well-being of medical students and there is little evidence on the role and impact of stress specifically on naturopathic medical students. The objective of this study was to review the available literature on strategies shown to improve the well-being of medical students. This literature review will guide the design of a prospective matched cohort study planned for the future to evaluate the impact of a longitudinal wellness program on mood and perceived stress of naturopathic medical students.

Methods: The authors searched Pubmed, Cochrane, Medline and Web of Science for peer reviewed, English-language articles using a combination of the following search terms: "medical students", "wellbeing", "strategies", "tools", "coping", "support" and "stress management". The search targeted studies which focused on well-being strategies implemented by medical schools between the time period of date of database inception to November 30th, 2019.

Results: 797 articles were found in total and 15 were identified which met the inclusion criteria for review including: 7 randomized controlled trials, 6 qualitative studies, and 2 quantitative studies. Studies collected data either via interviews, small group meetings, essays, questionnaires, and surveys. Interventions included: stress management workshops, resilience training, mindfulness meditation and reflective practice. Most studies found that students in intervention groups experienced lower levels of psychological distress and perceived stress, as well as, an improvement in general well-being, mindfulness, resilience, academic performance and better coping strategies. Results were sustained when studies included follow up, and students recommended well-being focused programs. 
UNDERGRADUATE RESEARCH IN NATURAL AND CLINICAL SCIENCE AND TECHNOLOGY (URNCST) JOURNAL Read more URNCST Journal articles and submit your own today at: https://www.urncst.com

Conclusion: The evidence for effectiveness of incorporating wellness focused programs and strategies into the medical school curriculum is clear based on the studies which have been analyzed. Although the interventions and outcome measures varied, the common theme of students benefiting from wellness strategies was prevalent. Further studies are warranted to develop curriculum changes allowing similar programs to be accessible to all medical students.

The authors have no conflict of interest to declare.

Effects of short term fasting on cancer treatment: A narrative review Yousef Sadat Nejad, CCNM Student [1], Sanam Arora, CCNM Student [1], Rick Bhim, ND [1], Sukriti Bhardwarj, ND [1] [1] Canadian College of Naturopathic Medicine, Toronto, Ontario, Canada

Background: It is estimated that 26,900 Canadian women will be diagnosed with breast cancer in 2019. Chemotherapy, a common treatment for breast cancer, uses cytotoxic drugs to destroy cancer cells which also destroy healthy cells. Chemotherapy is often associated with many side effects including, decreased quality of life, nausea and vomiting, fever, fatigue, and infection. Multiple randomized control trials have shown that short term fasting (STF) during chemotherapy is well tolerated and improves quality of life, fatigue and toxicity. The purpose of this study was to review the data on short term fasting as adjuvant to chemotherapy.

Methods: PubMED was searched using a PICO strategy that was developed surrounding breast cancer, short term fasting, chemotherapy and safety with respect to toxity, DNA damage, quality of life and fatigue.

Results: Chemotherapy combined with a 24-72 hours fast compared to treatment chemotherapy alone significantly improved quality of life, assessed by the FACIT- measurement system (Quality of life questionnaire). Significant benefits of short-term fasting were noted, and all reported side effects were of low grade. Short term fasting before and after chemotherapy reduced hematological toxicity and may induce a faster recovery of DNA damage in peripheral blood mononuclear cells. Hematological toxicity was measured based on the level of Gamma-H2AX (shows breaks in double stranded DNA).

Conclusion: Preliminary data shows that STF is safe but challenging in cancer patients receiving chemotherapy. In clinical studies, STF emerges as a promising strategy to enhance the efficacy and tolerability of chemotherapy. It appears safe as adjuvant to chemotherapy in humans, and it may reduce side effects and DNA damage in healthy cells in response to chemotherapy. However, more research is needed to firmly establish clinical efficacy and safety.

The authors have no conflict of interest to declare.

Different health professionals' uses of strontium: A narrative review Brittany Harris, DCHM, CCNM Student [1], Paul Richard Saunders, PhD, ND [1-3]

[1] Canadian College of Naturopathic Medicine, Toronto, Ontario, Canada

[2] National University of Health Sciences Lombard, Illinois, United States of America

[3] Canadian College of Homeopathic Medicine, Toronto, Ontario, Canada

Background: The western medical system, naturopathic and homoeopathic paradigms seem contradictory, but the professions overlap. One overlap is strontium: each discipline uses a different strontium salt. Strontium ranelate (a prescription salt), strontium citrate (a dietary supplement), and strontium carbonate (a homoeopathic remedy). Here, strontium's use in western medicine, naturopathy, and homoeopathy is compared to illuminate the similarities in the professions and the differences.

Methods: The literature on ranelate, citrate and carbonate was reviewed using PubMed and Cochrane. Eight homoeopathic journals, multiple materia medicas and repertories from the nineteenth through the twenty-first century were also reviewed.

Results: 922 ranelate, 89 citrate, and 290 carbonate sources were found. While literature about carbonate dates back to 1831 , literature on citrate and ranelate didn't emerge until the late twentieth century. However, ranelate quickly amasses more studies than the others combined. Almost none of the homoeopathic sources are freely available, unlike ranelate and citrate abstracts.

Discussion: Important differences in data for strontium ranelate, citrate and carbonate exist. The plethora of ranelate data versus citrate data forces those who use citrate to make generalizations from ranelate to citrate. Homoeopathic data are collected on humans and document effects on every body-system, preventing generalizations from biomarkers or in-vitro studies. Homoeopathic texts from the 1800's document strontium's toxic effects on the cardiovascular system, an effect that likely contributed to ranelate's discontinuation in 2017. Citrate and ranelate literature focuses heavily on researching mechanisms of action, allowing for the development of new drugs/supplements and enquiry into new indications. Despite these differences, all three professions use strontium for a variety of bone pathologies.

Aucoin et al. | URNCST Journal (2020): Volume 4, Issue 3

Page A6 of A10

DOI Link: https://doi.org/10.26685/urnest.186 
UNDERGRADUATE RESEARCH IN NATURAL AND CLINICAL SCIENCE AND TECHNOLOGY (URNCST) JOURNAL Read more URNCST Journal articles and submit your own today at: https://www.urncst.com

Conclusion: Even though medical, homoeopathic, and naturopathic doctors differ in their philosophies of practice, they have discovered the osteogenic effects of strontium. Strontium reminds us that these professions are not diametrically opposed as is believed.

No funding was received for this project, there are no conflicts of interest to report.

The potential therapeutic effect of topical cannabinoids in psoriasis: A review of the literature Adrienne Elgie, CCNM Student [1], Adam Gratton, ND [1]

[1] Canadian College of Naturopathic Medicine, Toronto, Ontario, Canada

Background: Psoriasis is a common chronic, inflammatory dermatological disease characterized by skin lesions with disrupted keratinocytes. Despite a number of pharmacological interventions available, achieving total clearance is difficult and the side effect profiles of many of the available interventions is extensive. Several endocannabinoid system (ECS) receptors have been identified to play a role in dermal proliferation, differentiation and immune activation. In particular, phytocannabinoids (PCs), such as $\Delta$ 9-tetrahydrocannabinol (THC) and cannabidiol (CBD) from Cannabis sativa and $C$. indica have been shown to interact with the ECS suggesting potential therapeutic use. The purpose of this study was to evaluate the therapeutic potential of topical PCs by reviewing the available evidence.

Methods: A literature search was conducted on PubMed using the terms "cannabinoids" AND "psoriasis". A total of 23 articles were found.

Results: Animal studies have largely focused on describing the downstream effects of CB1 and CB2 receptor signaling which does not entirely explain the potential anti-psoriatic mechanism. Endocannabinoids have also been shown to interact with PPAR- $\gamma$ receptors and agonists of these receptors have been shown to inhibit proliferation of epithelial cells. A small trial using a CBD enriched ointment in humans suggests a statistically significant improvement in PASI scores with 90 days of treatment. THC has been shown to bind to CB1 receptors with high affinity and can also activate PPAR- $\gamma$ receptors, CBD does not activate CB1 or CB2 but decreases helper T-cell activity, as well as, IFN- $\gamma$ and IL-17, and cannabinol binds CB2 receptors with high affinity.

Conclusion: Much is still unknown about the role of the ECS in dermal health and human data is scarce. Animal data and a small human trial suggests that topical PCs may be beneficial in reducing the dermatological manifestations of psoriasis. More research is needed to fully capture the potential side effects of this therapy and determine whether these effects are sustained beyond 90 days.

The authors have no conflicts of interest to declare.

The potential therapeutic effect of topical cannabinoids in atopic dermatitis: A review of the literature LaToya Lewis CCNM Student [1], Adam Gratton ND [1] [1] Canadian College of Naturopathic Medicine, Toronto, Ontario, Canada

Background: Atopic dermatitis (AD) is a chronic relapsing-remitting inflammatory dermatological disorder characterized by itching and dermal barrier dysfunction and breakdown. Aside from the newer biologics developed, there have been few advances in therapeutic options for AD. Endocannabinoid receptors have been shown to be expressed in the skin and on immune cells suggesting a potential therapeutic effect. Animal and in vitro data suggests that modifying the endocannabinoid system (ECS) may be of therapeutic significance. Phytocannabinoids (PCs) from Cannnabis species plants interact with the endocannabinoid system (ECS) in a similar way to endocannabinoids and may prove useful for patients with AD. The purpose of this study was to evaluate the potential effect of topical phytocannabinoids by looking at available research.

Methods: A literature search was conducted on the PubMed and Cochrane databases using various combinations of the following search terms: "cannabinoid receptor", "atopic dermatitis", "eczema", "pruritis", "histamine", "mast cells" and "endocannabinoid system". All types of studies were included and a total of 8 studies summarized in this review.

Results: A study using a topical endocannabinoid reuptake inhibitor gel in dogs found benefit over vehicle after 22 days. Human trials suggest co-administration of topical cannabinoid products with corticosteroids are superior to corticosteroids alone. The PC THC has been shown to bind with high affinity to CB1 receptors in addition to activating PPAR $-\gamma$ receptors. Cannabinol can interact with CB2 receptors and CBD may have benefits through other mechanisms.

Discussion: Animal studies have looked at the symptom of itch, rather than focussing on AD as a disease entity. Both CB1 and $\mathrm{CB} 2$ receptor activity appear to reduce itch in $\mathrm{AD}$ models with $\mathrm{CB} 1$ activity increasing sebum production in dry skin, and $\mathrm{CB} 2$ activity interfering with mast cell activation and degranulation. 
UNDERGRADUATE RESEARCH IN NATURAL AND CLINICAL SCIENCE AND TECHNOLOGY (URNCST) JOURNAL Read more URNCST Journal articles and submit your own today at: https://www.urncst.com

Conclusion: Topical administration of PCs has not been adequately studied in humans. More research is needed to fully capture the potential side effects of this therapy given the psychoactive potential for some cannabinoids and the higher incidence of $\mathrm{AD}$ in the pediatric population.

The authors have no conflicts of interest to declare.

Dietary counseling in schizophrenia spectrum disorders: Creation and evaluation of an evidence-informed psychoeducational tool

Monique Aucoin BMSc, ND [1], Laura LaChance MD [2-3], Kieran Cooley ND [1,4]

[1] Canadian College of Naturopathic Medicine, Toronto, Ontario, Canada

[2] McGill University, Montreal, Quebec, Canada

[3] Centre for Addiction and Mental Health, Toronto, Ontario, Canada

[4] Australian Research Centre in Complementary and Integrative Medicine, University of Technology, Ultimo, New South

Wales, Australia

Objective: Diet has been identified as a modifiable risk factor for schizophrenia spectrum disorders; however, clinical use of dietary modification remains limited. A 2018 scoping review identified all of the scientific literature on this topic. Using the results of the review, this project's objective is to create and evaluate an evidence-based psychoeducational tool for front-line clinicians and patients to use in implementing dietary recommendations as part of clinical treatment of psychotic disorders, along with a brief clinician guidance document.

Methods: The tool creation and evaluation will be guided by a number of strategies, theoretical frameworks and established recommendations for psychoeducational tools. In order to ensure that this tool meets the needs of clinicians and patients, we will undertake a process to evaluate the tool through two focus groups with clinicians (four) and individuals with lived experience (six to eight). Feedback will be gathered to gage if the tool is helpful and capture any obstacles, challenges or recommendations. The sessions will be audio recorded with participant permission for review and thematic, inductive analysis. Recordings will be transcribed and analysed by two investigators. The tool will be revised according to feedback and a brief second interview with all participants will allow for feedback on the revised version.

Results and Conclusions: This project is in progress. The initial draft of the tool will be presented.

\author{
Patterns of integrative medicine (IM) use and its effects on quality of life of patients undergoing radiotherapy: A \\ protocol \\ Evrim Tezcanli Tjon A Meeuw, CCNM Student [1], Monique Aucoin, ND [1] \\ [1] Canadian College of Naturopathic Medicine, Toronto, Ontario, Canada
}

Background: Integrative oncology is the use of complementary treatments together with conventional protocols. Although research on integrative oncology has been growing, the potential effects of these treatments are underestimated as many patients remain hesitant to disclose integrative medicine (IM) treatments to their oncologists. This study aims to evaluate the use of integrative treatments of cancer patients undergoing radiotherapy and its effects on quality of life during the treatment course.

Methods: This questionnaire-based prospective study is being carried out at the Radiation Oncology Department of Health Sciences University in Istanbul. This study started in February 2020 and targets to enroll 90 patients receiving radiation treatment. Patients starting radiotherapy are eligible to participate if they speak Turkish. A 25 question, multiple-choice questionnaire was designed to assess patients' use and expectations from IM and whether they informed their oncologists. Additionally, the Turkish version of the European Organization for Research and Treatment of Cancer-Quality of Life Questionnaire (EORTC QLQ-C30 v.3.0) is being administered to participants before and after the completion of radiotherapy in order to evaluate global quality of life changes during treatment.

Results: IM use during radiotherapy will be evaluated in regards to participants' use of IM modalities, socio-demographic profile, cancer related characteristics (including the site of disease and their disease status) and quality of life changes during the course of treatment. The questionnaire results will be analyzed using SPSS and a p-value $<0.05$ will be considered as statistically significant.

Conclusion: This monocentric study will shed light on the prevalence and patterns of IM use during radiotherapy, as well as patients' willingness to disclose these treatments to their oncologists. It may provide insight into the potential effects of IM on quality of life. Based on the findings of this study we can work towards an integrated model of health-care provision and design further research on integrative approaches.

Conflict of Interest: The authors have no conflict of interest.

Aucoin et al. | URNCST Journal (2020): Volume 4, Issue 3

DOI Link: https://doi.org/10.26685/urnest.186

Page A8 of A10 
UNDERGRADUATE RESEARCH IN NATURAL AND CLINICAL SCIENCE AND TECHNOLOGY (URNCST) JOURNAL Read more URNCST Journal articles and submit your own today at: https://www.urncst.com

The relationship between serum vitamin $D$ status and breast cancer risk and occurrence: A narrative review Tehsina Jaffer, BA, CNP, CCNM student [1], Hanieh Vedadi, BSc, ND [1]

[1] Canadian College of Naturopathic Medicine, Toronto, Ontario, Canada, M2K 1E2

Introduction: Breast cancer is the most commonly diagnosed cancer in women worldwide, comprising 2,088,849 new cases in 2018 , accounting for approximately $25.4 \%$ of all newly diagnosed cancers in women. Globally, it is the fifth most common cause of cancer-related death in women. Vitamin D is essential for optimal function of the mammary glands. Low serum vitamin $\mathrm{D}$ is becoming more common and has been associated with breast cancer. This literature review investigates current evidence surrounding vitamin $\mathrm{D}$, its relationship with breast cancer and whether serum levels are associated with diagnosis in both pre- and post-menopausal women.

Methods: We utilized PubMed, Medline and ClinicalKey databases to conduct a search using the terms "breast cancer" and "serum vitamin D". We limited our search to meta-analyses and systematic reviews published within the last ten years $(2009$ - 2019). We included literature that studied the relationship between serum vitamin D levels and breast cancer risk and occurrence.

Results: Fifty-nine articles were reported by the two systematic reviews and two meta-analyses selected. These articles consist of observational studies, which encompass cross-sectional, cohort and case-control studies. The authors of the systematic reviews and meta-analyses concluded there is an inverse association between serum 25-hydroxy vitamin D concentration and breast cancer risk and occurrence. In addition, the authors concluded that vitamin D deficiency is prevalent in patients diagnosed with breast cancer.

Conclusion: The two systematic reviews and two meta-analyses analyzed suggest that serum 25-hydroxy vitamin D deficiency conveys a direct relation to both breast cancer risk and occurrence in pre- and post-menopausal women. However, there are inconsistencies in Vitamin D deficiency thresholds, with values ranging from $<10$ to $<32 \mathrm{ng} / \mathrm{mL}$. One limitation of this study was that it only included systematic reviews and meta-analyses, which may have resulted in the exclusion of relevant evidence found in other types of studies. Further research is necessary to clarify the role of vitamin D and its relationship with breast cancer.

\section{Conflicts of Interest}

The authors declare that they have no conflict of interests.

\section{Authors' Contributions}

MA and KC co-founded the first CCNM Research Day.

$\mathrm{MA}, \mathrm{CC}, \mathrm{VC}$ and $\mathrm{KC}$ contributed equally to planning the research competition, assisted in the collection and review of the abstract submissions, as well as support for authors selected for the competition while producing their posters.

\section{Acknowledgements}

Special thanks to Julia Zander, Digital Media Specialist at the Canadian College of Naturopathic Medicine. Her patience, and keen, creative eye, were key to the creation of research posters for this competition. We would also like to acknowledge the work of our poster judging panel for their critical appraisal and scientific discussion on the day of the competition.

\section{Funding}

Funding for this conference has been supported by Biotics Research Inc. through an arms-length donation to the Canadian College of Naturopathic Medicine's Student Innovation Fund as well as an anonymous donor.

\section{Article Information}

Managing Editor: Jeremy Y. Ng

Peer Reviewers: The abstracts were peer-reviewed by the research day's planning committee.

Article Dates: Received Mar 06 20; Published Mar 1320

\section{Citation}

Please cite this article as follows:

Aucoin M, Cardozo V, Chung C, Cooley K. The 4th Annual CCNM Research Day: Student Research \& Innovation in Naturopathic Medicine. URNCST Journal. 2020 Mar 13: 4(3). https:/urncst.com/index.php/urncst/article/view/186 DOI Link: https://doi.org/10.26685/urncst.186 
UNDERGRADUATE RESEARCH IN NATURAL AND CLINICAL SCIENCE AND TECHNOLOGY (URNCST) JOURNAL

Read more URNCST Journal articles and submit your own today at: https://www.urncst.com

\section{Copyright}

(C) Monique Aucoin, Valentina Cardozo, Christine Chung, Kieran Cooley. (2020). Published first in the Undergraduate Research in Natural and Clinical Science and Technology (URNCST) Journal. This is an open access article distributed under the terms of the Creative Commons Attribution License (https://creativecommons.org/licenses/by/4.0/), which permits unrestricted use, distribution, and reproduction in any medium, provided the original work, first published in the Undergraduate Research in Natural and Clinical Science and Technology (URNCST) Journal, is properly cited. The complete bibliographic information, a link to the original publication on http://www.urncst.com, as well as this copyright and license information must be included.

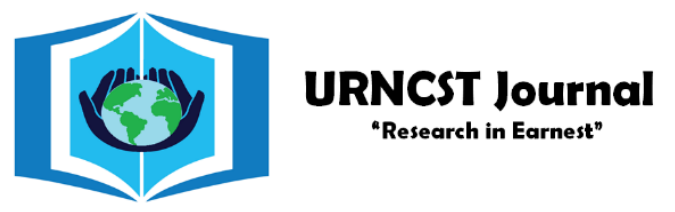

\section{Funded by the Government of Canada}

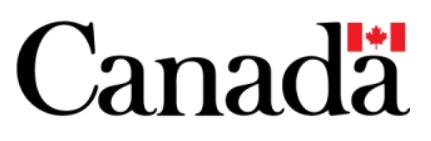

Do you research in earnest? Submit your next undergraduate research article to the URNCST Journal!

| Open Access | Peer-Reviewed | Rapid Turnaround Time | International | | Broad and Multidisciplinary | Indexed | Innovative | Social Media Promoted |

Pre-submission inquiries? Send us an email at info@urncst.com | Facebook, Twitter and LinkedIn: @URNCST

Submit YOUR manuscript today at https://www.urncst.com! 